\title{
Luc Leguérinel
}

Université Pédagogique de Cracovie, Pologne

http://dx.doi.org/10.18778/8088-896-8.22

\section{MINORITÉS SEXUELLES ET LIBERTINAGE DANS L'OEUVRE D'EVDOKIA NAGRODSKAÏA}

Depuis les écrits de Michel Foucault sur l'Histoire de la sexualité, nous savons que les images et discours de la sexualité sont culturellement construits, notamment avec sa distinction de deux procédures historiques sur la vérité du sexe. On retrouve la première dans l'ars erotica à Rome, ainsi qu'au sein des sociétés orientales et arabo-musulmanes où la vérité est extraite du plaisir en tant que tel, comme pratique et expérience sexuelles elles-mêmes. Le plaisir est pris en compte pour lui-même et comme ce qui doit être à connaître, comme un savoir réservé à la seule pratique sexuelle et qui doit demeurer secret pour ne pas perdre de son efficacité ni de sa vertu. La seconde, que l'on retrouve dans les pays occidentaux, se rapproche beaucoup plus d'une scientia sexualis, qui s'est développée durant des siècles pour dire la vérité du sexe à partir d'une forme de « pouvoir-savoir $\gg$, littéralement opposée à l'art de l'initiation et au secret magistral, puisqu'il est ici question de l'aveu qui est devenu « une des techniques les plus hautement valorisées pour produire le vrai $\gg^{1}$. En effet, depuis le Moyen Age on attend de l'aveu à ce qu'il soit une production de vérité, volontaire ou extorquée par la torture afin d'obtenir une confession. Il a ensuite évolué pour se répandre dans toute la société, aussi bien dans la justice, la médecine, la pédagogie que dans les rapports familiaux, les relations amoureuses, ou dans l'ordre le plus quotidien, où chacun doit confesser face à l'autre, ses crimes, ses péchés, ses pensées et ses désirs... Tout en sachant, bien évidemment, que le sexe fut depuis la pénitence chrétienne jusqu'aujourd'hui la matière préférée de la confession.

Dans son étude, Foucault nous montre que tout en étant un rituel de discours où le sujet qui parle coïncide avec le sujet de l'énoncé, l'aveu « est aussi un rituel qui se déploie dans un rapport de pouvoir, car on n'avoue pas sans la présence au moins virtuelle d'un partenaire qui n'est pas simplement l'interlocuteur, mais l'instance qui requiert l'aveu, l'impose, l'apprécie et intervient pour juger, punir, pardonner, consoler, réconcilier $\gg^{2}$. Autrement dit, cette forme, avec ces vérités avouées, est au plus loin de celle des initiations savantes au plaisir qui régit

1 Cf. Michel Foucault, Histoire de la sexualité I, Gallimard, Paris, 1970, p. 79.

2 Ibidem, p. 83. 
《l'art érotique », car nous appartenons à une société qui a ordonné le savoir du sexe, non plus dans la transmission du secret, mais autour de la lente montée de la confidence.

En rompant avec les traditions de l'ars erotica, notre société s'est donné une scientia sexualis, qui a poursuivi la tâche de produire des discours vrais sur le sexe, ajustant l'ancienne procédure de l'aveu sur les règles du discours scientifique. La scientia sexualis, développée à partir du $\mathrm{XIX}^{\mathrm{e}}$ siècle, garde paradoxalement pour noyau le rite singulier de la confession obligatoire et exhaustive, qui fut dans l'Occident chrétien la première technique pour produire la vérité du sexe. Ce rite, depuis le $\mathrm{XVI}^{\mathrm{e}}$, s'est peu à peu détaché du sacrement de pénitence, pour émigrer vers la pédagogie, les relations familiales, la médecine et la psychiatrie, qui ont permis de produire sur le sexe des discours vrais, à travers « un dispositif qui enjambe largement l'histoire puisqu' il branche la vieille injonction de l'aveu sur les méthodes de l'écoute clinique $»^{3}$. C'est aussi au sein de ce dispositif « qu'a pu apparaitre comme vérité du sexe et de ses plaisirs quelque chose comme la 'sexualité' $\gg^{4}$, qui s'est également développée dans la littérature.

Toutefois si cela est vrai pour l'Occident ça l'est beaucoup moins pour la Russie, car ce principe de vases communicants permettant une transition complexe entre les discours religieux et la littérature ne pouvait tout simplement pas fonctionner de la même manière, parce que l'Eglise orthodoxe russe n'a rien développé d'analogue à la confession catholique. Comme le remarque Alexei Lalo, « la Russie manque de modèles pour les récits de l'interdit et la culture de la culpabilité qui occupaient alors les clercs catholiques. Les échanges d'idées sur le corporel et son statut moral qui ont lieu entre les artistes, les lettrés et les intellectuels d'un côté et le folklore religieux de l'autre, se sont limités à la fascination de l'ancien avec des pratiques sexuelles souvent étranges dans certaines sectes populaires qui ont proliféré en Russie de la fin du XIX jusqu'au début du XX $\mathrm{XX}^{\mathrm{e}}$ siècle $\gg^{5}$. Autrement dit, ce sont les sectes religieuses, et non la science ou l'Église orthodoxe, qui ont développé de nouveaux discours sexuels en Russie, mais en tant que discours charnels, et non sur la sexualité dans la société. La condition préalable à ces débats provient de l'engagement des intellectuels dans les débats de l'époque, à la fois sur la modernisation et l'occidentalisation de la culture russe. Mais il faut attendre les intellectuels russes de l'Âge d'argent pour que se révèle un discours engagé sur la sexualité, sur lequel les représentations de classe et d'identité se jouent à l'aide de ressources héritées du passé et de façon différente des discours occidentaux à partir desquels de nouveaux problèmes et questions sociales sont

${ }^{3}$ Ibidem, p. 91.

${ }^{4}$ Ibidem.

${ }_{5}^{5}$ A. Lalo, Libertinage in Russian Culture and Littérature, Leidon-Boston, Brill, 2011, p. 2. 
arrivés dans le pays. En d'autres termes s'il ne semble pas faire de doute que la littérature occidentale a bien influencé cette avant-garde intellectuelle russe de l'ère prérévolutionnaire, il n'empêche qu'il faut voir les variantes russes traitant des questions qui touchent à la sexualité et à l'érotisme comme des produits culturels originaux, et non pas comme un dérivé de l'Ouest, bien qu'ils y répondent. En effet, si elle s'inspirait des écrits occidentaux, cette avant-garde ne se limitait ni ne dérivait d'eux en aucune manière, car ces écrits russes étaient envisagés dans le champ russe de la production intellectuelle et totalement adaptés au contexte russe.

La Révolution russe de 1905 a permis de produire une censure nettement plus clémente que la précédente qui limitait les différents débats sexuels aux seules revues médicales. C'est dans ce contexte qu'est apparue la « jeune génération » de l'Âge d'argent, avec des écrivains qui ont utilisés des scénarios sexuels peu orthodoxes pour aborder la question de l'intensification du rôle de l'individu. On retrouve entre autres parmi ces écrivains Mihail Kuzmin qui publia Les ailes en 1906. Son roman décrit la germination psychologique d'un jeune homme qui, au-delà de la morale traditionnelle, cherchait à trouver le bonheur dans les bras d'autres hommes, qui lui firent prendre conscience de la sensualité, de la beauté de la vie, de la liberté indispensable à l'épanouissement de la personne humaine. C'était la première fois en Russie qu'un écrivain publiait un roman ouvertement homosexuel, suscitant un véritable scandale auprès de la critique conservatrice qui considérait cette œuvre comme rien d'autre que pornographique, alors que pour nombre d'autres lecteurs ce roman était vu comme une véritable libération à propos d'un sujet qui demeurait tabou. Une autre fiction homosexuelle est également apparue un an plus tard avec le roman Trente-trois monstres de Lidia Zinovieva-Annibal, qui fera scandale et sera censuré dès sa parution pour quelques temps. Ce livre est publié sous la forme d'un journal intime d'une femme et adressé à une autre du nom de Véra. La jeune femme est séduite à la veille de ses noces par Véra, une brillante et belle actrice, qui l'entraîne dans le lesbianisme et une vie demi-mondaine. Au début, soumise et manquant de confiance en elle, elle est promue comme actrice de second ordre, puis, devant sa beauté, Véra la fait poser pour trente-trois peintres qui se l'arrachent, pour trente-trois portraits adultères. Tourmentée par l'idée de fugacité de la beauté et désireuse de donner au monde son aimée, Véra la donne à ces hommes comme un sacrifice inéluctable, mais à la fin elle ne le supporte plus et en meurt. On peut percevoir dans ce livre l'antagonisme entre la beauté dominatrice et la médiocrité soumise, il y a également une ambiguité entre l'activité de modèle et la prostitution, qui, du coup, paraît être une libération sexuelle bien plus que de la prostitution. La même année Mikhail Artsybachev publia son plus célèbre roman, Sanine qui causa un immense scandale et fut censuré dès sa publication, conduisant même son auteur à prendre le chemin de l'émigration pour Varsovie. Sanine, le héros principal de ce roman, est à n'en pas douter un homme libre, peut-être même un véritable stir- 
nérien, invitant au renversement des valeurs établies, avec une perpétuelle ironie, un cynisme qui révolte les conformistes et les réactionnaires. Individualiste et athée, il refuse de s'assujettir à quelque préjugé que ce soit, souhaitant que tous s'affranchissent de la mal-vie, du qu'en-dira-t-on, et jouissent franchement de la beauté du monde, et évoque la jouissance en termes crus et vrais, de manière poétique, au-delà des carcans religieux et moraux. Les livres de ces trois auteurs ont été traduits en langue française, et sont relativement connus d'un public averti. Or il en va différemment pour le roman d'Evdokia Nagrodskaïa, Le courroux de Dionysos, qui fut publié en 1910 et connut dès sa sortie un succès en librairies, au point d'avoir été réédité une dizaine de fois en l'espace de six ans, et d'avoir été traduit en plusieurs langues et probablement en français, sans que l'on puisse en avoir la certitude parce que ce livre est aujourd' hui totalement introuvable et s'il reste peu de trace de l'auteure à travers le monde, il n'en reste quasiment plus aucune en France. Pourtant son nom était devenu l'un des plus célèbres dans le monde littéraire dans la Russie de l'époque.

Il existe peu d'informations concernant Nagrodskaïa, si ce n'est qu'elle est née à Saint-Pétersbourg en 1866 et qu'elle fut élevée dans un environnement intellectuel. Fille unique d'Avdotia Panaïeva (1819-1893), femme de lettres russe, auteure prolifique de romans qu'elle publia sous le pseudonyme de N. Stanitsky et de Mémoires, et d'Apollon Golovachev le second époux d'Avdotia, qui travaillait comme journaliste dans la revue politico-littéraire Le Contemporain (Sovremennik). Avdotia Panaïeva avait, avant cela, été l'épouse de l'écrivain Ivan Panaïev, collaborateur de l'écrivain politique et poète Nikolaï Nekrassov dans Le Contemporain dont elle fut également la maitresse durant quinze ans et avec qui elle écrivit deux romans. Ils vivaient ensemble dans le même appartement et formaient un atypique couple à trois ${ }^{6}$. La mère d'Evdokia était aussi connue pour l'éminent salon pétersbourgeois qu'elle tenait, et où l'on pouvait notamment rencontrer des écrivains tels que Belinski, Dostoïevski, Dobrolioubov, Tourgueniev, Herzen, et beaucoup d'autres?

Il est clair que l’atmosphère dans laquelle Evdokia Nagrodskaïa a été élevée, a contribué au développement de ses valeurs et idées, même s'il semble qu'à l'aube de sa vie adulte, elle préférait le culte de la domesticité à celui de Dionysos. A commencer par son premier mariage en 1882, à 17 ans, avec le prince Bek Melik Tangiev dont on ne connait rien si ce n'est qu'il fut hussard dans une milice caucasienne, et peut-être les quelques lignes qu'elle trace dans Le courroux de Diony-

${ }^{6}$ Cf. Ruth «Sobel, Avdot'ia Iakovlevna Panaeva 1819-1893 », [in] Reference Guide to Russian Littérature, Editor Neil Cornwell, Associate Editor Nicole Christian, Fitzroy Dearborn Publishers, London-Chicago, 1998, p. 611-612.

7 Cf. Awdotia Panajewa (Gołowaczowa), Wspomnienia, Wydawnictwo Literackie, Kraków, 1977. 
sos, lorsque Tatiana, le personnage principal du roman, parle de son premier mari qu'elle avait rencontré dans le salon de sa mère et avec qui elle se maria à l'âge de 17 ans avant de devenir veuve, sans grand regret, trois ans plus tard, parce qu'il avait pensé pouvoir rembourser ses dettes avec la dot de sa femme et qu'il l'avait insultée en retournant six mois après son mariage à ses anciennes amours. Pour sa part, Nagrodskaïa, suite à son veuvage en janvier 1888, retourna s'installer à Saint-Pétersbourg chez sa mère avec ses deux enfants. C'est à cette époque qu'elle fit ses débuts en tant qu'écrivain avec son récit Le cadeau de Noël, paru dans la revue L'étoile (Звезаа). Toutefois, par souci alimentaire, elle a dû se tourner vers une littérature populaire en publiant notamment des romans policiers tels que L'affaire noire, Noud coulant et Les secrets de Pétersbourg en complément dans le journal Le monde (Cвem) des années 89/90, ou encore en publiant des nouvelles « pour dames » sur la solitude, l'incompréhension et le caractère illusoire dans les pages de grandes revues, comme Le bonheur perplexe (Живописное обозрение 1892. №16), Les roses et Sous les saules (Север. 1892. №19,32). Mais Nagrodskaïa ne trouve vraiment son sujet littéraire qu'à partir de son roman $\mathrm{La}$ fille du diable, où elle peint le portrait d'une mystérieuse femme fatale qui, au final, se révèle être pleine de bonté 8 .

Elle épouse en 1896, à l'âge de 31 ans, Vladimir Nagrodski qui est alors ingénieur dans les chemins de fer et président du conseil d'administration de deux sociétés russes de chemins de fer, avant d'enseigner en 1911 à l'Institut des voies de communication à Saint-Pétersbourg. C'est sous ce nouveau nom qu'Evdokia fait ses débuts, après dix ans de silence, avec son roman Le courroux de Dionysos. Comme nous l'avons déjà signalé, ce roman a apporté à son auteure une large célébrité. Il s'appuie sur des conceptions philosophiques importantes pour l'époque, à commencer par le titre qui fait directement référence à Nietzsche et au concept nietzschéen de l'homme-artiste qui intériorise une lutte entre le rationalisme apollinien et la passion dionysienne, mais son roman fait également référence au livre Sexe et caractère d'Otto Weininger, qui propose une formule pour calculer l'identité sexuelle, en montrant que chaque individu possède les caractéristiques des deux sexes et que la question clé de la vie moderne est de les maintenir en équilibre. Nous pouvons enfin retrouver dans ce livre des thématiques freudiennes sur l'inconscient, les besoins primaires et l'inversion sexuelle. Ces philosophes abordaient le problème de changements importants et offraient des explications à un public russe désorienté, qui avait raison de se sentir abandonné par une intelligentsia hautaine vis-à-vis de son choix littéraire, sans offrir au-

8 Cf. О. A. Kuzniecowa, Pусскал митература XX века. Прозаики, поэты, драматурги. Биобиблиографический словарь. Том 2. 3 - O. с. 599-600. (Littérature Russe du XX siècle. Prosateurs, poètes, dramaturges. Dictionnaire bibliographique, volume 2.3 - O., c. 599-600.) 
cune alternative satisfaisante pour répondre à ses besoins particuliers. C'est aussi pourquoi les lecteurs de l'époque avaient confiance en les auteurs de littérature populaire qui, comme Nagrodskaïa, tout en se référant à certaines conceptions philosophiques sans pour autant les mentionner, pouvaient contribuer à la reconstruction de la société, en aidant leurs lecteurs à atteindre des niveaux plus élevés de sophistication, nécessaire à la création d'une société capable de gérer le stress psychologique et technologique de la modernisation, de façon à aider et à remodeler la réalité sociale?.

Le courroux de Dionysos fait référence au titre d'une peinture réalisée par l'héroïne du roman, Tatiana Kuznetsova. En tant qu'artiste, Tatiana est une figure culturelle accomplie dans une société qui les privilégiait de la même façon que l'élite intellectuelle. En effet, les artistes, tout comme les intellectuels étaient glorifiés en Russie dans leur dévouement pour le bien commun, en s'exprimant à travers leur art. C'est d'ailleurs en ce sens que les préoccupations de Tatiana pour l'achèvement de son chef-d'œuvre pouvaient être comprises en termes politiques, comme si elle était impliquée dans un mouvement de réforme sociale. Son statut lui permettait également de vivre en dehors des conventions morales normales, en étant à la fois veuve, concubine et maîtresse. Malgré un fond assez mince, l'histoire de ce roman est un riche mélange de politique culturelle et d'exploration sur l'identité sexuelle. Il commence par le voyage de Tatiana vers le sud de la Russie, anxieuse de rencontrer la famille d'Ilya, l'homme avec qui elle a vécu dans le bonheur en tant que concubine. Dans le train, elle se retrouve de manière incontrôlée et érotiquement attirée par Edgar Stark, un homme d'affaires anglais qui est de tout point de vue à l'opposé d' Ilya. Ils se séparent avant que leur attraction sexuelle réciproque n'explose, mais elle devient obsédée par Stark. Quand ils se croisent de nouveau, elle est finalement impuissante contre ses désirs. Sans qu'Ilya ait connaissance de leur liaison, elle se rend à Rome pour terminer sa peinture, en utilisant Stark comme modèle pour Dionysos. L'attraction de Tatiana pour Stark et son besoin de l'exprimer à travers sa peinture font partie du même problème psychologique ; cela devient clair pour elle quand, après avoir terminé sa peinture, elle se lasse de Stark parce que leur relation était purement physique. Elle aspire à retourner auprès d'Ilya pour retrouver une vie de contemplation intellectuelle et de respect mutuel, mais elle se découvre enceinte de Stark. Bien qu'après l'accouchement elle laisse son fils à Stark, afin de pouvoir rester avec Ilya, l'émotion de l'amour maternel qu'elle ressent lui complique un peu plus la vie. Avec cet enfant, se pose pour l'hérö̈ne un problème émotionnel qui délimite une lutte constante entre un idéal de vie et la réalité, entre le choix et la maternité et en ce sens Nagrodskaïa ramène Tatiana au sens de ses obligations

${ }^{9}$ L. McReynolds, Introduction to The Wrath of Dionysus, Bloomington, Indiana University Press, 1997, p. XIII. 
personnelles. Mais, Tatiana est une femme ayant des traits traditionnellement attribués aux hommes, comme le lui reproche d'ailleurs Stark, en l'accusant d'une certaine façon d'être seulement attirée par son corps, et non pas de tout son être qui l'aime profondément et très tendrement ${ }^{10}$. Elle-même ne se rend pas tout à fait compte de cette anomalie, jusqu'à ce que son ami Latchinov, lui-même homosexuel non déclaré, en lui avouant qu'il est également amoureux de Stark, lui explique qu'elle est en fait un « homme avec le corps d'une femme », en ajoutant qu'elle aurait dû être lesbienne ${ }^{11}$. La « théorie Latchinov » qui au premier abord inquiète Tatiana, contribue également à une union paisible, entre son concubin et son amant efféminé et leur fils. Tatiana accepte enfin un ménage mensonger à trois comme seul équilibre possible pour toutes les personnes impliquées. Et bien qu'en tant qu'intellectuelle russe, la vérité est son but le plus élevé, dans le cas présent, l'artiste-hérö̈ne célèbre la morale du dieu romain de l'érotisme et de l'hédonisme qui est une véritable antithèse de la Russie intellectuelle et conteste toute supériorité morale de cette dernière. Douloureusement consciente que seul un compromis peut aplanir les problèmes qu'elle a causés à ceux qu'elle aime, et que ces concessions sont au coût de ses principes, l'héroïne choisit finalement d'en payer un prix en compromettant ses normes d'intellectuelle russe. Ce faisant, elle soulève des doutes quant à savoir jusqu'où les idéaux de l'intelligentsia peuvent être appliqués à la vie réelle.

Mais le message de Nagrodskaïa est un peu plus complexe que cet aperçu l'a indiqué : alors que Latchinov est clairement un homosexuel dont la vie a été ruinée par son incapacité à se déclarer ouvertement, Tatiana est une « nouvelle femme », pas dans le sens où elle est nécessairement une féministe ou une lesbienne refoulée, mais parce que l'auteure la présente comme engagée dans un renversement des rôles traditionnels homme - femme. En effet, dès le début du roman Tatiana est décrite en termes masculins, comme une femme très rationnelle, de sang-froid, résolue et pleine $\mathrm{d}>$ initiative ; alors que les caractéristiques féminines de Stark servent de complément à ce qui apparaît comme son alter ego masculin, étant de belle apparence, bien que faible, hystérique et passif. Le roman se termine avec Latchinov rappelant à Tatiana qu'elle était néanmoins une $\ll$ femme chanceuse $\gg^{12}$.

Les lecteurs russes des années 1910 étaient eux-mêmes différents de la génération précédente comme ce le fut pour Nagrodskaïa, ce qui explique la montée rapide de sa popularité. La révolution de 1905 avait aiguisé la question de la place de l'individu dans une société industrielle. Et si Nagrodskaïa est représentative

10 E. Nagrodskaïa, The Wrath of Dionysus: A Novel, Bloomington, Indiana University Press, 1997, p. 114.

11 Ibidem, p. 182-183.

12 Ibidem, p. 191. 
des écrivains méprisés par l'intelligentsia qui considérait le roman populaire tout juste bon pour des femmes de classe moyenne avec des goûts peu raffinés et des prétentions à la haute couture, il n'empêche qu'elle répondait aux besoins de cette lecture publique émergente, en donnant à la société russe des clés pour penser par elle-même. Les critiques lui reprochaient également son « extrémisme » féministe et son caractère tendancieux avec l'inversion sexuelle de ses personnages et des motifs homosexuels jamais loin de la surface. En effet, si les tensions sexuelles explorées dans ce roman ne portent pas sur les ardeurs physiques de Tatiana et de ses amants, elles portent plutôt sur ce qui détermine les identités sexuelles des différents personnages. Bien que l'ensemble des accouplements décrit dans le livre soit hétérosexuel, ce roman est essentiellement homosexuel, mais il s'agit d'une homosexualité latente. Les personnages principaux de Nagrodskaïa semblaient plus inversés que pervertis, selon les normes de l'époque. De ce fait, son incursion dans l'homosexualité diffère fondamentalement de celle de Kuzmin et de Zinoveva-Annibal, car dans le roman de Nagrodskaïa un seul des personnages accomplit ses désirs de même sexe, et bien que l'auteur le dépeigne avec bienveillance, le personnage lui-même se déteste pour son orientation sexuelle et se révèle incapable de l'assumer. Nagrodskaïa rend cette question moins menaçante pour les lecteurs, en encadrant son histoire autour de la recherche d'une persona sexuelle qui pouvait permettre un accouplement contre nature paradoxalement naturel d'un homme et d'une femme, et dès que le masculin et le féminin avaient réprimé leur véritable nature homosexuelle, ils pouvaient se satisfaire les uns des autres.

Cela n'est pas le cas de son deuxième roman, La porte de Bronze (1911), qui a attiré l'ire des censeurs avec les multiples déviations sexuelles de la norme. Elle y présente un artiste homosexuel opposé à sa sœur dans leur rivalité pour l'affection d'un autre homme. Le thème de La porte dans le titre est directement tiré de Freud; dans ses rêves d'enfance, l'artiste se transforme en femme quand il va derrière cette mystérieuse porte de bronze. Peu de temps après la publication les censeurs ont confisqué toutes les copies. Deux ans plus tard, Nagrodskaïa publie une seconde version, plus tempérée, rebaptisée À la Porte de Bronze. L'héroïne très masculine d'un autre roman, Les Mauvais Esprits (1915), suggère le lesbianisme. Ce roman montre également certains signes de l'intérêt que Nagrodskaïa porte à l'occultisme, qui se rapproche à l'intérêt de l'époque pour l'inconscient. Cet engouement pour le surnaturel rend évident les angoisses populaires concernant le rationnel et le monde technologique créé par l'industrialisation.

Aucune de ses œuvres ultérieures ne pouvait atteindre la popularité du Courroux de Dionysos, mais le corpus des travaux de Nagrodskaïa fournit un baromètre culturel utile pour mesurer l'évolution des goûts et des intérêts du public à la fin de la Russie impériale, de l'intérêt le plus sincère pour le sexe à l'absorption du mysticisme et de notions de choix personnel qui sont apparus avec l'évolution de la société de consommation. Son roman La Colonnade Blanche (1914) utilise 
l'idée d'un symbole récurrent de l'anneau pour inspirer les personnages à prendre le contrôle de leur vie. L'hérö̈ne, Kitty, aperçoit une colonnade blanche en revenant de l'enterrement de son oncle. Brillante à travers la pluie sur le porche lointain, avec un escalier lui faisant signe vers le haut, la colonnade est là pour servir de symbole à sa conscience d'une vie meilleure. Elle lui apparait en période de stress, et l'encourage, par exemple, à se libérer de son fiancé aristocratique et égocentrique. Une foule de personnages secondaires en viennent également à réaliser qu'elle devrait prendre des mesures pour améliorer sa situation, et ceux qui voient la colonnade de Kitty peuvent commercer à avoir une vie meilleure. Un sous-thème familier revient avec la maîtresse-actrice du fiancé de Kitty, quand elle parle du lesbianisme, en voyant qu'il pourrait offrir une alternative à sa sexualité autodestructrice. Un des autres personnages décrit comment la colonnade a œuvré en sa faveur, en voyant clairement, qu'il peut y avoir quelque chose de mieux que les circonstances qui l'entourent ${ }^{13}$.

Nagrodskaïa a également publié plusieurs recueils de nouvelles, qui lui ont offert un forum littéraire à partir duquel elle pouvait exposer plus largement ses préoccupations sociales et intellectuelles. Notamment avec le personnage-titre dans Ania, qui se prostitue à un homme d'affaires dans le but de protéger la réputation de son père, qui avait contrefait des stocks d'argent pour les livrer à sa mấtresse, une danseuse espagnole bon marché. Peu à peu, Ania prend conscience qu'elle bénéficie d'une plus grande satisfaction de sa relation avec cet homme que celle qu'elle avait eu avec un père ingrat, et elle poursuit la liaison de son propre gré. En permettant à Ania de faire un choix et de sélectionner l'amant plutôt que la famille, Nagrodskaïa crée une nouvelle conception de fin heureuse. Lui, d'autre part, est purement une histoire de temps occulte et de voyage spatial, avec quelques éléments sur la psyché intérieure ou la psychologie sexuelle moderne. Le personnage acquiert des qualités humaines quand il sauve d'un asile d'aliénés une jeune fille qui était considérée comme folle pour ses fantastiques ébats amoureux avec lui. Nagrodskaïa fait également une descente inhabituelle dans les bas-fonds avec Le Cauchemar, cette histoire évoque les œuvres de Maxime Gorki et Alexander Kouprine. Cette description impudique de la dépravation sexuelle dans les bidonvilles diffère distinctement de sa confusion moderniste habituelle sur l'identité sexuelle.

En octobre 1918, Nagrodskaïa émigre avec son mari à Paris. Elle ne sera jamais active dans la politique des émigrés, mais elle poursuit son œuvre avec ses deux romans de 1922, La vérité sur la famille de ma femme et Mémoires de Romain Vassilieff. Mais son meilleur travail de l'avis des critiques, est sa trilogie historique Fleuve des temps (1924-26), qui est une réflexion sur la franc-maçonnerie comme perfectionnement moral spirituel. Cependant cette saga diffère trop de ses œuvres

${ }^{13}$ Cf. L. McReynolds, op. cit., p. XIV-XV. 
précédentes pour générer beaucoup d'attention. Les choses mystérieuses l'ayant toujours préoccupée, Nagrodskaïa devait subir - peut-être aussi sous l'influence de son mari ${ }^{14}$ - l'attraction de la franc-maçonnerie. Admise au Droit Humain et maître dans la loge Aurore elle devint une adepte, zélée, infatigable en l'étude des symboles de l'Art Royal. La loge russe Avdora lui doit aussi sa fondation et son caractère rigoureusement initiatique adapté à la mentalité slave. D'ailleurs le seul écrit dont il reste une trace d'elle en français est une pièce de théâtre La Dame et le Diable, qui fut publié en 1933, à titre posthume dans une revue maçonnique. Evdokia mourut trois ans plus tôt à l'âge de 63 ans, le 19 mai 1930 à Paris et fut inhumée au cimetière de Thiais.

Il est clair que si Nagrodskaïa est entrée dans la littérature populaire un peu plus tard que ses homologues occidentales, son œuvre mérite cependant de sortir de l'oubli dans lequeelle est plongée, car contrairement à l'avis des critiques de l'époque, généralement mal à l'aise avec la franchise sur le sexe de la littérature post-1905, et pour qui ce genre d'écrivains avait tendance à écrire de la « pornographie $\gg$, il faudrait plutôt $y$ voir une romancière avant-gardiste, traitant de libertinage beaucoup plus que de pornographie, car il existe bien pour Nagrodskaïa une combinaison de la libre-pensée sexuelle et politique, notamment avec son livre Le courroux de Dionysos. Pour reprendre Alain Badiou ${ }^{15}$ sur ce point, Tatiana annonce la venue d'une nouvelle femme, parce qu'elle construit un entre-deux, une place hors place, qui définit toute féminité en s'opposant à « la forte affirmation de l'Un, du pouvoir unique, qui caractérise la position masculine traditionnelle $\gg^{16}$. Cette logique du deux se retrouve tout au long du roman sous différents angles, entre concubin et amant, amour et désir, vérité et mensonge, mère et séductrice, hétérosexualité et homosexualité. Et en ce sens, Tatiana s'oppose à l'affirmation de l'Un, du pouvoir unique, qui caractérise l'homme dans la société traditionnelle. Déjà en tant que veuve elle prouve qu'elle a rencontré la médiation sexuelle d'un homme et supprime ainsi le premier symbole fondamental qui est celui de la virginité dans les sociétés traditionnelles, et en tant que mère elle laisse aussi à Stark le soin de s'occuper au début de son enfant. Elle s'y oppose également lorsqu'elle se sait enceinte et ne comprend pas pourquoi elle devrait se marier lorsque Stark le lui demande pour que l'enfant ne soit pas illégitime elle compre encore moins pourquoi elle devrait se marier à l'église, mais elle s'en moque et pour couper court à la conversation elle répond à Stark : « Tu peux

14 Vladimir Nagrodski fut en effet l'un des fondateurs des loges Astrée et Hermès, et auteur d'écrits francs-maçons comme entre autres Initiation géométrique. Le Secret de la lettre G. Rose et Croix. Les Croix symboliques, en 1935.

15 A. Badiou, « Figures de la féminité dans le monde contemporain », conférence prononcée à Athènes, été 2010.

16 Ibidem, p. 14-18. 
m'épouser dans une église orthodoxe, anglicane, une synagogue, une pagode... une pyramide si tu veux $! \gg{ }^{17}$ Autrement dit, elle ne souhaite pas se laisser réduire à la logique du mariage, ni devenir mère par la médiation du mariage, et donc par la médiation d'un homme dont la logique se résume dans l'unité absolue du Nom-du-Père. Or comme le montre Badiou, «le symbole de cette unité absolue est du reste évident dans l'unité absolue, et absolument masculine, du Dieu des grands monothéismes. Or, c'est de cet Un qu'il est question, de façon critique, dans l'entre-deux figural où se tient une femme $\gg^{18}$. Et en ce sens, c'est comme un phénomène littéraire moderne ou de modernisation que Le courroux de Dionysos devrait être pris. Il faut bien dire de modernisation car à la fin du roman, après la mort d'Ilya, Tatania accepte de se marier avec Stark plus de dix ans après la naissance de leur fils, mais à l'hôtel de ville et dans deux églises, Orthodoxe et Anglicane. Comme si ces deux églises symbolisaient à elles-seules, la reprise du deux sur l'Un absolu d'un Dieu réellement mort, même s'il est vrai que nous ne cessons encore d'être encombrés de son cadavre.

\section{Sexual minorities and libertinism in the works of Evdokia Nagrodskaia}

The author aims to present the work of Evdokia Nagrodskaia, a Russian author now fallen into oblivion. To do this, he briefly discusses the literary context of the time and her biography, before analyzing in greater depth her book The Wrath of Dionysus, which was a bestseller in Russia in the early 20th century. He tries, through the analysis of this novel, to rediscover a novelist both despised by the Russian intelligentsia for writing popular literature, and strongly criticized by the former censors for treating themes related both to female adultery, homosexuality and lesbianism. By that he's trying to show that Nagrodskaia is primarily a novelist avant-garde which deals with libertinism far more than pornography and that her freethinking is both sexual and political.

Keywords : the Silver Age, Russian writer, libertinism, adultery, homosexuality, lesbianism. Mots clés : l'âge d’argent, écrivain russe, libertinage, adultère, homosexualité, lesbianisme.

17 E. Nagrodskaïa, op. cit., p. 119.

18 A. Badiou, ibidem. 